\title{
A Finite and Instantaneous Screw Based Approach for Topology Design and Kinematic Analysis of 5-Axis Parallel Kinematic Machines
}

Tao Sun ${ }^{1 *}$, Shuo-Fei Yang ${ }^{1}$, Tian Huang ${ }^{1,2}$ and Jian S. Dai ${ }^{3}$

\begin{abstract}
Unifying the models for topology design and kinematic analysis has long been a desire for the research of parallel kinematic machines (PKMs). This requires that analytical description, formulation and operation for both finite and instantaneous motions are performed by the same mathematical tool. Based upon finite and instantaneous screw theory, a unified and systematic approach for topology design and kinematic analysis of PKMs is proposed in this paper. Using the derivative mapping between finite and instantaneous screws built in the authors' previous work, the finite and instantaneous motions of PKMs are analytically described by the simple and non-redundant screws in quasi-vector and vector forms. And topological and parametric models of PKMs are algebraically formulated and related. These related topological and parametric models are ready to do type synthesis and kinematic analysis of PKMs under the unified framework of screw theory. In order to show the validity of the proposed approach, a kind of two-translational and three-rotational (2T3R) 5-axis PKMs is taken as example. Numerous new structures of the 2T3R PKMs are synthesized as the results of topology design, and their Jacobian matrix is obtained easily for parameter optimization and performance evaluation. Some of the synthesized PKMs have outstanding capabilities in terms of large workspaces and flexible orientations, and have great potential for industrial applications of machining and manufacture. Among them, METROM PKM is a typical example which has attracted a lot of attention from global companies and already been developed as commercial products. The approach is a general and unified approach that can be used in the innovative design of different kinds of PKMs.
\end{abstract}

Keywords: Innovative design, Parallel kinematic machines, Screw theory, Finite screw, Instantaneous screw

\section{Introduction}

$\mathrm{CNC}$ and robot based equipment are important parts to push "Made in China 2025" plan [1, 2]. In comparison with traditional CNCs and articulated robots, parallel kinematic machines (PKMs) are demonstrated by many researchers to have advantages of high stiffness, good accuracy, excellent dynamics and reconfigurability through deeply investigating their topology structures [3-5], stiffness characteristics [6], constraint properties [7] and kinematic performances [8-10]. They are suitable to be applied in machining and repairing of large scale

\footnotetext{
*Correspondence: stao@tju.edu.cn

${ }^{1}$ Key Laboratory of Mechanism Theory and Equipment Design of Ministry of Education, Tianjin University, Tianjin 300350, China

Full list of author information is available at the end of the article
}

component with complex surface. The innovative design of PKMs is always a hot topic and draws great attention from both academia and industry $[11,12]$.

The innovative design of PKMs usually consists of topology design and kinematic analysis [13-15]. It has long been a desire to unify these two parts under a framework of the same mathematical tool. This mathematical tool should be able to realize analytical description, formulation and operation of both finite and instantaneous motions, and then relate topological and parametric models algebraically. Till now, there are three available mathematical tools at hand, i.e., matrix group, dual quaternion and screw theory.

Matrix group was firstly given out by Lie and later utilized by Klein to describe rigid body motion. It was Hervé et al. $[16,17]$ who gave an approach to formulate
Springer Open

(c) The Author(s) 2018. This article is distributed under the terms of the Creative Commons Attribution 4.0 International License (http://creativecommons.org/licenses/by/4.0/), which permits unrestricted use, distribution, and reproduction in any medium, provided you give appropriate credit to the original author(s) and the source, provide a link to the Creative Commons license, and indicate if changes were made. 
topological models and carried out type synthesis of PKMs through describing finite motions of PKMs by subgroups of the matrix representation of the special Euclidean group SE(3) and their composite manifolds. Using this approach, many PKMs having different motions were synthesized by Li and Hervé $[18,19]$. Describing instantaneous motions by Lie algebra of SE(3), i.e., se(3), Brockett [20] applied the exponential mapping between $\mathrm{SE}(3)$ and se(3) to relating topological and parametric models of open-loop mechanisms. His work was extended to deal with closed-loop and other kinds of mechanisms. However, two barriers are encountered when using matrix groups for finite motion composition in formulating topological models. One barrier arises from that matrix group cannot directly reflect the Chasles' axis as well as the angular and/or linear displacement about and/or along that axis. Thus, the description of finite motion by matrix group is complicated. The other barrier comes from the incompetent to algebraically compute the finite motion composition result of matrix groups by using the Baker-Campbell-Hausdorff formula. Topological models of many PKMs cannot be simply written as the group products of a few Lie subgroups of SE(3). Hence, type synthesis of these PKMs cannot be precisely carried out although parametric models for kinematic analysis can be directly obtained by se(3).

Dual quaternion can be traced back to the early work of Euler, Rodrigues and Hamilton. Perez and McCarthy [21] are probably the first to use it in the finite and instantaneous motion analysis of serial kinematic chains. Unit dual quaternions and unit pure dual quaternions are used by them to respectively describe finite and instantaneous motions, because the algebraic structure of the former is a double cover of SE(3) whose Lie algebra in turn constitutes the latter. Using group theory, Selig [22] and Dai [23] investigated algebraic properties of the exponential and Cayley mappings between these two kinds of dual quaternions, resulting in a clear relationship between the finite and instantaneous models. It should be noted that even though both finite and instantaneous motions can be described by dual quaternions, quaternion representation is not the simplest form. The redundancy may cause complexity in analytical operations of finite motions. Additionally, the Rodrigues formula with dual angles is not the simplest form of the Baker-CampbellHausdorff formula in composition of finite motions.

Screw theory was firstly proposed by Ball and has been developed to be a powerful tool in analysis and mechanical design of PKMs. Instantaneous screw has been proved to be the simplest and most effective form to describe instantaneous motion and widely used in formulating parametric models to conduct velocity and force [24-26], precision [27, 28] and stiffness [29-32] analysis. In the authors' previous work [33-36], finite screw is proved to be the concise and non-redundant form to describe finite motion and can be analytically composited by the screw triangle product [33]. Meanwhile, the algebraic structures of these two kinds of screws were revealed and the derivative mapping between them was built by the authors [33]. All these achievements show that finite and instantaneous screw theory has the potential to unify topology design and kinematic analysis into a general and consistent process by doing type synthesis and kinematic analysis under this concise mathematical tool, which can overcome the shortcomings of the above matrix group and dual quaternion based approaches.

Mainly drawing on finite and instantaneous screw theory, this paper proposes a unified and systematic approach for topology design and kinematic analysis of PKMs. A kind of two-translational and three-rotational (2T3R) PKMs is taken as example to show the validity of the proposed approach. These PKMs generate twoDoF translations in a fixed plane followed by three-DoF rotations about a fixed point. Some of them have great potential for industrial applications in 5-axis machining and manufacture because of their outstanding capabilities to realize large workspaces and flexible orientations. METROM PKM is a typical one which has attracted a lot of attention from global companies and already been developed as commercial products [37, 38]. The approach is a general and unified approach that can be used in the innovative design of different kinds of PKMs.

The paper is organized as follows. Having a brief review of the state-of-the-art of the existing approaches based upon different mathematical tools to uniformly describe finite and instantaneous motions in Section 1, Section 2 presents the theoretical foundations of finite and instantaneous screw theory. The topological models of PKMs are formulated by describing the PKMs, their limbs and joints by finite screws in Section 3, and type synthesis of the 2T3R PKMs is done to show the usages and advantages of the formulated model. In Section 4, the parametric models of PKMs are directly obtained through differentiating the topological models, one typical structure of the 2T3R PKMs, i.e., the METROM PKM, is selected to show the detailed procedures. The conclusions of this paper are drawn in Section 5 (Additional file 1).

\section{Screw Theory: Finite and Instantaneous Screws}

In this section, we firstly introduce the basic concepts and properties of finite and instantaneous screws, which lays the theoretical foundations of type synthesis and kinematic analysis for innovative design of PKMs.

According to the Chasles's theorem and Mozzi's theorem, both finite motion and instantaneous motion of a rigid body can be regarded as a rotation about an axis followed by a translation long that axis, as shown in Figures 1 and 2. 


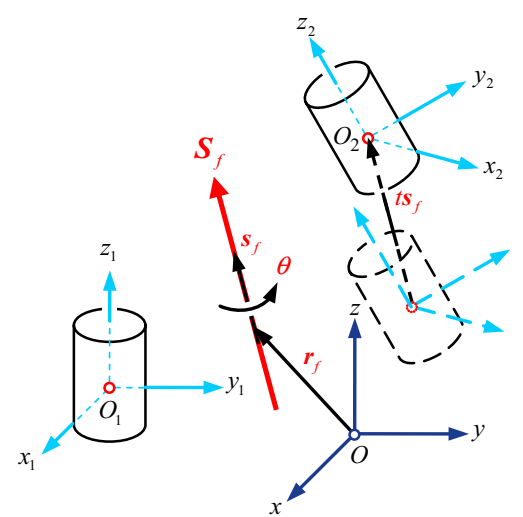

Figure 1 Finite motion of a rigid body

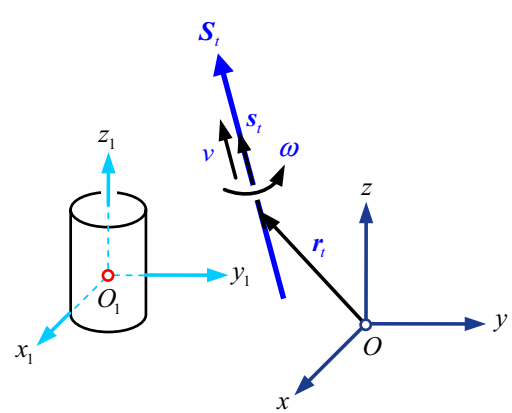

Figure 2 Instantaneous motion of a rigid body

For finite motion, the axis is referred as Chasles's axis. A finite motion is a pose (including orientation and position) transformation of a rigid body from its initial pose to arbitrary pose about and along Chasles's axis. It can be expressed by a finite screw in quasi-vector form [33] in the simplest and non-redundant manner as

$$
\boldsymbol{S}_{f}=2 \tan \frac{\theta}{2}\left(\begin{array}{c}
\boldsymbol{s}_{f} \\
\boldsymbol{r}_{f} \times \boldsymbol{s}_{f}
\end{array}\right)+t\left(\begin{array}{c}
\mathbf{0} \\
\boldsymbol{s}_{f}
\end{array}\right),
$$

where $\boldsymbol{s}_{f}$ and $\boldsymbol{r}_{f}$ denote the unit vector and position vector of the Chasles' axis, $\theta$ and $t$ are the rotational angle about and translational distance along that axis with respect to the initial pose.

For instantaneous motion, the axis is referred as Mozzi's axis. An instantaneous motion is a rigid body velocity measured at a given pose, which is constituted by angular velocity about and linear velocity along Mozzi's axis. For simplicity, it is usually expressed by an instantaneous screw (twist) in vector form [39-41] as

$$
\boldsymbol{S}_{t}=\omega\left(\begin{array}{c}
\boldsymbol{s}_{t} \\
\boldsymbol{r}_{t} \times \boldsymbol{s}_{t}
\end{array}\right)+v\left(\begin{array}{c}
\mathbf{0} \\
\boldsymbol{s}_{t}
\end{array}\right),
$$

where $\boldsymbol{s}_{t}$ and $\boldsymbol{r}_{t}$ denote unit vector and position vector of the Mozzi's axis, $\boldsymbol{\omega}$ and $\boldsymbol{v}$ are angular velocity about and linear velocity along that axis.

As is well known, successive finite screws of a rigid body from its initial pose to final pose via several intermediate poses should be composited in nonlinear manner. The composition of any two finite screws is expressed by

$$
\begin{aligned}
\boldsymbol{S}_{f, a b} & =\boldsymbol{S}_{f, a} \Delta \boldsymbol{S}_{f, b}=\left(\boldsymbol{S}_{f, a}+\boldsymbol{S}_{f, b}+\frac{\boldsymbol{S}_{f, b} \times \boldsymbol{S}_{f, a}}{2}\right. \\
& \left.-\tan \frac{\theta_{a}}{2} \tan \frac{\theta_{b}}{2}\left(t_{b}\left(\begin{array}{c}
\mathbf{0} \\
\boldsymbol{s}_{f, a}
\end{array}\right)+t_{a}\left(\begin{array}{c}
\mathbf{0} \\
\boldsymbol{s}_{f, b}
\end{array}\right)\right)\right) / \\
& \left(1-\tan \frac{\theta_{a}}{2} \tan \frac{\theta_{b}}{2} \boldsymbol{s}_{f, a}^{\mathrm{T}} \boldsymbol{s}_{f, b}\right),
\end{aligned}
$$

where

$$
\begin{aligned}
& \boldsymbol{s}_{f, a}=2 \tan \frac{\theta_{a}}{2}\left(\begin{array}{c}
\boldsymbol{s}_{f, a} \\
\boldsymbol{r}_{f, a} \times \boldsymbol{s}_{f, a}
\end{array}\right)+t_{a}\left(\begin{array}{c}
\mathbf{0} \\
\boldsymbol{s}_{f, a}
\end{array}\right), \\
& \boldsymbol{s}_{f, b}=2 \tan \frac{\theta_{b}}{2}\left(\begin{array}{c}
\boldsymbol{s}_{f, b} \\
\boldsymbol{r}_{f, b} \times \boldsymbol{s}_{f, b}
\end{array}\right)+t_{b}\left(\begin{array}{c}
\mathbf{0} \\
\boldsymbol{s}_{f, b}
\end{array}\right) .
\end{aligned}
$$

$S_{f, a}$ and $S_{f, b}$ are two arbitrary successive finite screws generated by the same rigid body, the symbol " $\Delta$ " is referred to as screw triangle product and is proven by the authors of this paper in Ref. [33].

Using the screw triangle product in Eq. (3), the resultant finite screw of the rigid body from its initial pose to final pose can be obtained through computing screw triangle products of all the successive finite screws it generates during its continuous finite motion.

Unlike the nonlinear composition of finite screws, instantaneous screws are composited in linear way. Suppose a rigid body generates two velocities at a given pose. Each is expressed by an instantaneous screw as

$$
\begin{aligned}
& \boldsymbol{S}_{t, a}=\omega_{a}\left(\begin{array}{c}
\boldsymbol{s}_{t, a} \\
\boldsymbol{r}_{t, a} \times \boldsymbol{s}_{t, a}
\end{array}\right)+v_{a}\left(\begin{array}{c}
\mathbf{0} \\
\boldsymbol{s}_{t, a}
\end{array}\right), \\
& \boldsymbol{S}_{t, b}=\omega_{b}\left(\begin{array}{c}
\boldsymbol{s}_{t, b} \\
\boldsymbol{r}_{t, b} \times \boldsymbol{s}_{t, b}
\end{array}\right)+v_{b}\left(\begin{array}{c}
\mathbf{0} \\
\boldsymbol{s}_{t, b}
\end{array}\right) .
\end{aligned}
$$

The resultant instantaneous screw can be obtained by adding them together

$$
S_{t, a b}=S_{t, a}+S_{t, b} .
$$

As proved in our previous work [33], arbitrary finite screw that a rigid body generates from its initial pose can be written in the form shown in Eq. (1). Regarding its initial pose as the given pose, arbitrary instantaneous screw the rigid body generates has the form in Eq. (2). At the initial pose $(\theta=0$ and $t=0)$ where the Chasles' axis is coincident with the Mozzi's axis at the instant, there 
exists differential mapping between finite and instantaneous screws. This means the derivative of finite screw is derived to be instantaneous screw [33]

$$
\begin{aligned}
\left.\dot{\boldsymbol{S}}_{f}\right|_{\theta=0} & =\dot{\theta}\left(\begin{array}{c}
\boldsymbol{s}_{f} \\
\boldsymbol{r}_{f} \times \boldsymbol{s}_{f}
\end{array}\right)+\dot{t}\left(\begin{array}{c}
\mathbf{0} \\
\boldsymbol{s}_{f}
\end{array}\right) \\
& =\omega\left(\begin{array}{c}
\boldsymbol{s}_{t} \\
\boldsymbol{r}_{t} \times \boldsymbol{s}_{t}
\end{array}\right)+v\left(\begin{array}{c}
\mathbf{0} \\
\boldsymbol{s}_{t}
\end{array}\right)=\boldsymbol{S}_{t} .
\end{aligned}
$$

This property leads to the algebraic structures of finite and instantaneous screws. The entire set of finite screws forms a Lie group under screw triangle product, while the entire set of instantaneous screws is the corresponding Lie algebra under screw cross product. The underlying relationship between these two kinds of screws is thus revealed.

Using this relationship, the topology design and kinematic analysis of PKMs can be integrated into the unified framework of screw theory. This is because:

(1) The topological model of a PKM can be formulated by describing finite motions of the PKM, its limbs and joints utilizing finite screws.

(2) The parametric model of the PKM can be directly obtained through differentiating its topological model at given pose.

(3) Type synthesis and kinematic analysis for innovative design of PKMs can be easily carried out using these two models under the simple and consistent screw theory.

In the following two sections, 5-axis PKMs having 2T3R motion will be taken as example to show the detailed procedures of this finite and instantaneous screw based approach for topology design and kinematic analysis. Firstly, type synthesis of this kind of PKMs will be done utilizing finite screws, which will result in numerous new topology structures with potential industrial applications. Then, the kinematic analysis of a typical structure, i.e., the METROM PKM, will be conducted to show how to directly obtain the parametric model based upon instantaneous screws through differentiating the corresponding topological model. The Jacobian matrix and constraint force will be formulated, which is ready for parameter optimization and performance evaluation.

\section{Type Innovative Design Based upon Finite Screws}

\subsection{Topological Model of a PKM}

Suppose a PKM is composed of $l$ open-loop limbs, as shown in Figure 3. Each limb consists $n_{i}(i=1,2, \cdots, l)$ one-DoF joints (revolute joints $(\mathrm{R})$ and prismatic joints

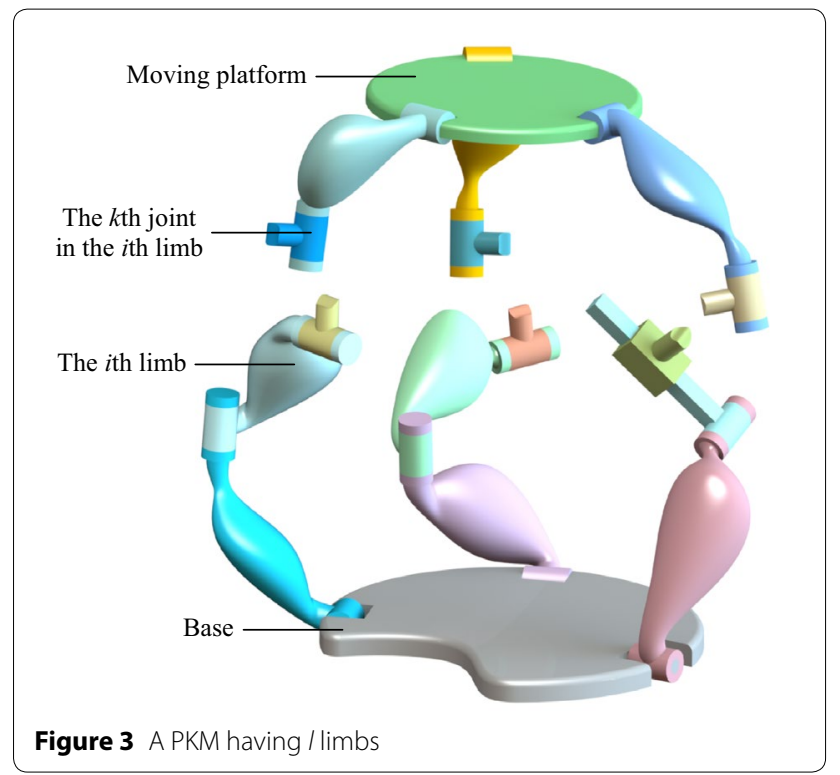

$(\mathrm{P}))$. Because the finite motions of the PKM's moving platform can be obtained as the intersection of those of its limbs, and the finite motions of each limb are the composition of those of all joints in it, following analytical equations can be formulated through describing the finite motions of the PKM, its limbs and joints as finite screws.

$$
\begin{aligned}
& \boldsymbol{S}_{f, \mathrm{PKM}}=\boldsymbol{S}_{f, 1} \cap \boldsymbol{S}_{f, 2} \cap \cdots \cap \boldsymbol{S}_{f, l}, \\
& \boldsymbol{S}_{f, i}=\boldsymbol{S}_{f, i, n_{i}} \Delta \boldsymbol{S}_{f, i, n_{i}-1} \triangle \cdots \Delta \boldsymbol{S}_{f, i, 1}, i=1,2, \cdots, l,
\end{aligned}
$$

$\boldsymbol{S}_{f, i, k}=\left\{\begin{array}{cc}2 \tan \frac{\theta_{i, k}}{2}\left(\begin{array}{c}\boldsymbol{s}_{i, k} \\ \boldsymbol{r}_{i, k} \times \boldsymbol{s}_{i, k}\end{array}\right), & \mathrm{R} \text { joint, } \\ t_{i, k}\left(\begin{array}{c}\mathbf{0} \\ \boldsymbol{s}_{i, k}\end{array}\right), \quad \text { P joint, }\end{array} k=1,2, \cdots, n_{i}\right.$.

Where $S_{f, \text { PKM }}$ denotes the finite screw generated by the PKM, $S_{f, i}$ is the finite screw generated by its $i$ th limb, $S_{f, i, k}$ is the finite screw of the $k$ th joint in the $i$ th limb. The denotations of $\boldsymbol{s}_{i, k}, \boldsymbol{r}_{i, k}, \theta_{i, k}$ and $t_{i, k}$ can be referred to the symbols in Eq. (1).

Equations (6)-(8) contain all the topological information of the PKM, including:

(1) The number of limbs and the number of joints in each limb;

(2) The type of each joint, i.e., $R$ joint or $P$ joint;

(3) The direction and position of each joint, i.e., the geometrical arrangement of each joint in the limb which it belongs to;

(4) The geometrical relationships among different limbs. 
Thus, these three equations can exactly serve as topological model of the PKM, which can be used for topology design. In what follows, we take 5-Axis PKMs having 2T3R motion as example to show the usage of this finite screw based topological model in doing type synthesis, resulting in numerous new topology structures with potential industrial applications.

\subsection{Type Synthesis of 2T3R 5-Axis PKMs}

The main goal of type synthesis is inventing innovative mechanisms with new topology structures having the given motion pattern. The expected motion pattern of the discussed PKMs is 2T3R, i.e., two-DoF translations in a fixed plane followed by three-DoF rotations about a fixed point $O$, which allows the PKMs realize 5-axis machining. Hence, the finite motions of a PKM with this 2T3R motion can be written using finite screw as

$$
\begin{array}{r}
\boldsymbol{S}_{f, \mathrm{PKM}}=t_{2}\left(\begin{array}{c}
\mathbf{0} \\
\boldsymbol{s}_{2}
\end{array}\right) \Delta t_{1}\left(\begin{array}{c}
\mathbf{0} \\
\boldsymbol{s}_{1}
\end{array}\right) \Delta 2 \tan \frac{\theta_{c}}{2}\left(\begin{array}{c}
\boldsymbol{s}_{c} \\
\boldsymbol{r}_{O} \times \boldsymbol{s}_{c}
\end{array}\right) \\
\Delta 2 \tan \frac{\theta_{b}}{2}\left(\begin{array}{c}
\boldsymbol{s}_{b} \\
\boldsymbol{r}_{O} \times \boldsymbol{s}_{b}
\end{array}\right) \Delta 2 \tan \frac{\theta_{a}}{2}\left(\begin{array}{c}
\boldsymbol{s}_{a} \\
\boldsymbol{r}_{O} \times \boldsymbol{s}_{a}
\end{array}\right) .
\end{array}
$$

According to Eq. (6), the feasible limb structures for the 2T3R PKMs should generate finite screws that contain $\boldsymbol{S}_{f, \mathrm{PKM}}$ in Eq. (9)

$$
\boldsymbol{S}_{f, \mathrm{PKM}} \subseteq \boldsymbol{S}_{f, i}
$$

Through adding none or one translational factor into $S_{f, \mathrm{PKM}}$, finite screws of the feasible limb structures should have either of the following two expressions

$$
\begin{aligned}
& \boldsymbol{S}_{f, i}=\boldsymbol{S}_{f, \mathrm{PKM}}, \\
& \boldsymbol{S}_{f, i}=\boldsymbol{S}_{f, \mathrm{PKM}} \Delta t_{3}\left(\begin{array}{c}
\mathbf{0} \\
\boldsymbol{s}_{3}
\end{array}\right),
\end{aligned}
$$

which correspond to the 5-DoF and 6-DoF limb structures, respectively.

Substituting Eq. (10) into Eqs. (11) and (12) leads to the two standard $S_{f, i}$

$$
\begin{aligned}
\boldsymbol{s}_{f, \mathrm{~L}_{\mathrm{I}}} & =t_{2}\left(\begin{array}{c}
\mathbf{0} \\
\boldsymbol{s}_{2}
\end{array}\right) \Delta t_{1}\left(\begin{array}{c}
\mathbf{0} \\
\boldsymbol{s}_{1}
\end{array}\right) \Delta 2 \tan \frac{\theta_{c}}{2}\left(\begin{array}{c}
\boldsymbol{s}_{c} \\
\boldsymbol{r}_{O} \times \boldsymbol{s}_{c}
\end{array}\right) \\
& \Delta 2 \tan \frac{\theta_{b}}{2}\left(\begin{array}{c}
\boldsymbol{s}_{b} \\
\boldsymbol{r}_{O} \times \boldsymbol{s}_{b}
\end{array}\right) \Delta 2 \tan \frac{\theta_{a}}{2}\left(\begin{array}{c}
\boldsymbol{s}_{a} \\
\boldsymbol{r}_{O} \times \boldsymbol{s}_{a}
\end{array}\right), \\
\boldsymbol{s}_{f, \mathrm{~L}_{\mathrm{II}}} & =t_{2}\left(\begin{array}{c}
\mathbf{0} \\
\boldsymbol{s}_{2}
\end{array}\right) \Delta t_{1}\left(\begin{array}{c}
\mathbf{0} \\
\boldsymbol{s}_{1}
\end{array}\right) \Delta 2 \tan \frac{\theta_{c}}{2}\left(\begin{array}{c}
\boldsymbol{s}_{c} \\
\boldsymbol{r}_{O} \times \boldsymbol{s}_{c}
\end{array}\right) \\
& \Delta 2 \tan \frac{\theta_{b}}{2}\left(\begin{array}{c}
\boldsymbol{s}_{b} \\
\boldsymbol{r}_{O} \times \boldsymbol{s}_{b}
\end{array}\right) \Delta 2 \tan \frac{\theta_{a}}{2}\left(\begin{array}{c}
\boldsymbol{s}_{a} \\
\boldsymbol{r}_{O} \times \boldsymbol{s}_{a}
\end{array}\right) \Delta t_{3}\left(\begin{array}{c}
\mathbf{0} \\
\boldsymbol{s}_{3}
\end{array}\right),
\end{aligned}
$$

where $\boldsymbol{S}_{f, \mathrm{~L}_{\mathrm{I}}}$ and $\boldsymbol{S}_{f, \mathrm{~L}_{\mathrm{II}}}$ denote the first and second standard $\boldsymbol{S}_{f, i}$

According to Eqs. (9) and (10), joint types and arrangements of the limb structures which generate the above two expressions can be obtained. Thus, Eqs. (13) and (14) correspond to two standard limbs, $\mathrm{R}_{a} \mathrm{R}_{b} \mathrm{R}_{c} \mathrm{P}_{1} \mathrm{P}_{2}$ and $\mathrm{P}_{3} \mathrm{R}_{a} \mathrm{R}_{b} \mathrm{R}_{c} \mathrm{P}_{1} \mathrm{P}_{2}$, where the subscripts denote the directions of the joints. Based upon these two standard limbs, all the derivative limbs can be synthesized using the properties of screw triangle product. It should be noted that we only concern the 5-DoF limbs here, because the 6-DoF ones can be easily obtained by random permutation of the joints in $\mathrm{P}_{3} \mathrm{R}_{a} \mathrm{R}_{b} \mathrm{R}_{c} \mathrm{P}_{1} \mathrm{P}_{2}$.

A 5-DoF derivative limb of $\mathrm{R}_{a} \mathrm{R}_{b} \mathrm{R}_{c} \mathrm{P}_{1} \mathrm{P}_{2}$ should satisfy the following two conditions:

(1) The finite screw it generates is equivalent to the standard one, i.e., $\boldsymbol{S}_{f, \mathrm{~L}_{\mathrm{I}}}$ in Eq. (13), and thus denoted as $\boldsymbol{S}_{f, \mathrm{~L}_{\mathrm{p}}}^{\prime}$

(2) The five factors in $\boldsymbol{S}_{f, \mathrm{~L}_{\mathrm{I}}}^{\prime}$ are the same with those in $\boldsymbol{S}_{f, \mathrm{~L}_{\mathrm{I}}}$ but have different sequence, or $\boldsymbol{S}_{f, \mathrm{~L}_{\mathrm{I}}}^{\prime}$ has different factor(s) with $\boldsymbol{S}_{f, \mathrm{~L}_{\mathrm{I}}}$.

Firstly, we consider the situation that $\boldsymbol{S}_{f, \mathrm{~L}_{\mathrm{I}}}$ and $\boldsymbol{S}_{f, \mathrm{~L}_{\mathrm{I}}}$ have the same five factors. It means that the corresponding derivative limb structures have the same five joints with $\mathrm{R}_{a} \mathrm{R}_{b} \mathrm{R}_{c} \mathrm{P}_{1} \mathrm{P}_{2}$. Hence, these derivative limb structures can be obtained by permutation of $\mathrm{R}_{a} \mathrm{R}_{b} \mathrm{R}_{c} \mathrm{P}_{1} \mathrm{P}_{2}$ while unchanging the finite screw it generated. Because the three $R$ joints constituted a spherical joint (S), their direction can be arbitrarily chosen. Thus, we can suppose that the direction of $R_{c}$ is perpendicular to the directions of $\mathrm{P}_{1}$ and $\mathrm{P}_{2}$, i.e., $\boldsymbol{s}_{c} \times\left(\boldsymbol{s}_{1} \times \boldsymbol{s}_{2}\right)=\mathbf{0}$. In this way, it can be proved that arbitrarily adjusting the sequence among $\mathrm{R}_{c}$, $\mathrm{P}_{1}$ and $\mathrm{P}_{2}$ will always result in the derivative limb structures that satisfy the two conditions.

For example, $\mathrm{R}_{a} \mathrm{R}_{b} \mathrm{P}_{1} \mathrm{P}_{2} \mathrm{R}_{c}$ can be obtained through changing the order of $\mathrm{R}_{c}$ in $\mathrm{R}_{a} \mathrm{R}_{b} \mathrm{R}_{c} \mathrm{P}_{1} \mathrm{P}_{2}$. Based upon Eqs. (7) and (8), $\boldsymbol{S}_{f, \mathrm{~L}_{\mathrm{I}}}^{\prime}$ generated by $\mathrm{R}_{a} \mathrm{R}_{b} \mathrm{P}_{1} \mathrm{P}_{2} \mathrm{R}_{c}$ can be formulated as

$$
\begin{aligned}
\boldsymbol{S}_{f, \mathrm{~L}_{\mathrm{I}}}^{\prime} & =2 \tan \frac{\theta_{c}}{2}\left(\begin{array}{c}
\boldsymbol{s}_{c} \\
\boldsymbol{r}_{O} \times \boldsymbol{s}_{c}
\end{array}\right) \Delta t_{2}\left(\begin{array}{c}
\mathbf{0} \\
\boldsymbol{s}_{2}
\end{array}\right) \Delta t_{1}\left(\begin{array}{c}
\mathbf{0} \\
\boldsymbol{s}_{1}
\end{array}\right) \\
& \triangle 2 \tan \frac{\theta_{b}}{2}\left(\begin{array}{c}
\boldsymbol{s}_{b} \\
\boldsymbol{r}_{O} \times \boldsymbol{s}_{b}
\end{array}\right) \Delta 2 \tan \frac{\theta_{a}}{2}\left(\begin{array}{c}
\boldsymbol{s}_{a} \\
\boldsymbol{r}_{O} \times \boldsymbol{s}_{a}
\end{array}\right) .
\end{aligned}
$$

Equation (15) can be rewritten as follows by computing the resultant of the first three factors using the screw triangle product in Eq. (3)

$$
\begin{aligned}
\boldsymbol{s}_{f, \mathrm{~L}_{\mathrm{I}}}^{\prime} & =2 \tan \frac{\theta_{c}}{2}\left(\begin{array}{c}
\boldsymbol{s}_{c} \\
\boldsymbol{r}_{O} \times \boldsymbol{s}_{c}+\frac{t_{1} \boldsymbol{s}_{1}+t_{2} \boldsymbol{s}_{2}}{2 \tan \frac{\theta_{c}}{2}}+\frac{\left(t_{1} \boldsymbol{s}_{1}+t_{2} \boldsymbol{s}_{2}\right) \times \boldsymbol{s}_{c}}{2}
\end{array}\right) \\
& \Delta 2 \tan \frac{\theta_{b}}{2}\left(\begin{array}{c}
\boldsymbol{s}_{b} \\
\boldsymbol{r}_{O} \times \boldsymbol{s}_{b}
\end{array}\right) \Delta 2 \tan \frac{\theta_{a}}{2}\left(\begin{array}{c}
\boldsymbol{s}_{a} \\
\boldsymbol{r}_{O} \times \boldsymbol{s}_{a}
\end{array}\right) .
\end{aligned}
$$


Equation (16) has the following equivalent expression for $\boldsymbol{s}_{c} \times\left(\boldsymbol{s}_{1} \times \boldsymbol{s}_{2}\right)=\mathbf{0}$,

$$
\begin{aligned}
\boldsymbol{s}_{f, \mathrm{~L}_{\mathrm{I}}}^{\prime} & =2 \tan \frac{\theta_{c}}{2}\left(\begin{array}{c}
\boldsymbol{s}_{c} \\
\boldsymbol{r}_{c} \times \boldsymbol{s}_{c}
\end{array}\right) \Delta 2 \tan \frac{\theta_{b}}{2}\left(\begin{array}{c}
\boldsymbol{s}_{b} \\
\boldsymbol{r}_{O} \times \boldsymbol{s}_{b}
\end{array}\right) \\
& \triangle 2 \tan \frac{\theta_{a}}{2}\left(\begin{array}{c}
\boldsymbol{s}_{a} \\
\boldsymbol{r}_{O} \times \boldsymbol{s}_{a}
\end{array}\right),
\end{aligned}
$$

where

$$
\boldsymbol{r}_{c}^{\prime}=\boldsymbol{r}_{O}-\frac{\left(t_{1} \boldsymbol{s}_{1}+t_{2} \boldsymbol{s}_{2}\right) \times \boldsymbol{s}_{c}}{2 \tan \frac{\theta_{c}}{2}}+\frac{t_{1} \boldsymbol{s}_{1}+t_{2} \boldsymbol{s}_{2}}{2} .
$$

Following the similar way to derive Eq. (17) from Eq. (15), Eq. (13) can be rewritten as

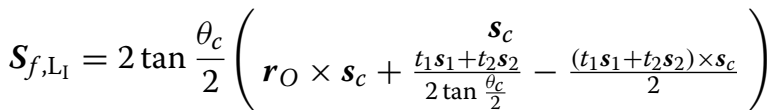

$$
\begin{aligned}
& \Delta 2 \tan \frac{\theta_{b}}{2}\left(\begin{array}{c}
\boldsymbol{s}_{b} \\
\boldsymbol{r}_{O} \times \boldsymbol{s}_{b}
\end{array}\right) \Delta 2 \tan \frac{\theta_{a}}{2}\left(\begin{array}{c}
\boldsymbol{s}_{a} \\
\boldsymbol{r}_{O} \times \boldsymbol{s}_{a}
\end{array}\right) \\
& =2 \tan \frac{\theta_{c}}{2}\left(\begin{array}{c}
\boldsymbol{s}_{c} \\
\boldsymbol{r}_{c} \times \boldsymbol{s}_{c}
\end{array}\right) \Delta 2 \tan \frac{\theta_{b}}{2}\left(\begin{array}{c}
\boldsymbol{s}_{b} \\
\boldsymbol{r}_{O} \times \boldsymbol{s}_{b}
\end{array}\right) \\
& \triangle 2 \tan \frac{\theta_{a}}{2}\left(\begin{array}{c}
\boldsymbol{s}_{a} \\
\boldsymbol{r}_{O} \times \boldsymbol{s}_{a}
\end{array}\right),
\end{aligned}
$$

where

$$
\boldsymbol{r}_{c}=\boldsymbol{r}_{O}-\frac{\left(t_{1} \boldsymbol{s}_{1}+t_{2} \boldsymbol{s}_{2}\right) \times \boldsymbol{s}_{c}}{2 \tan \frac{\theta_{c}}{2}}-\frac{t_{1} \boldsymbol{s}_{1}+t_{2} \boldsymbol{s}_{2}}{2} .
$$

Because $\left(t_{1} \boldsymbol{s}_{1}+t_{2} \boldsymbol{s}_{2}\right) \times \boldsymbol{s}_{c}$ and $t_{1} \boldsymbol{s}_{1}+t_{2} \boldsymbol{s}_{2}$ are two arbitrary orthogonal vectors that are perpendicular to $\boldsymbol{s}_{c}$, both $\boldsymbol{r}_{c}$ and $\boldsymbol{r}_{c}^{\prime}$ denote arbitrary vectors perpendicular to $\boldsymbol{s}_{c}$. Thus, Eq. (15) is equivalent to Eq. (13).

In this manner, two derivative limb structures, $\mathrm{R}_{a} \mathrm{R}_{b} \mathrm{P}_{1} \mathrm{P}_{2} \mathrm{R}_{c}$ and $\mathrm{R}_{a} \mathrm{R}_{b} \mathrm{P}_{1} \mathrm{R}_{c} \mathrm{P}_{2}$, can be synthesized.

Secondly, we consider the situation that $\boldsymbol{S}_{f, \mathrm{~L}_{\mathrm{I}}}^{\prime}$ has different factor(s) with $S_{f, \mathrm{~L}_{\mathrm{I}}}$. In this situation, each derivative limb structure has at least one different joint with $\mathrm{R}_{a} \mathrm{R}_{b} \mathrm{R}_{c} \mathrm{P}_{1} \mathrm{P}_{2}$. Supposing that the direction of $\mathrm{R}_{c}$ is perpendicular to that of $\mathrm{P}_{1}$, i.e., $\boldsymbol{s}_{c}^{\mathrm{T}} \boldsymbol{s}_{1}=0$, it can be proved that the generated finite screw will not be changed if we use one $\mathrm{R}_{c}$ to replace $\mathrm{P}_{2}$, or two $\mathrm{R}_{c}$ to replace $\mathrm{P}_{1}$ and $\mathrm{P}_{2}$.

For example, $\mathrm{R}_{a} \mathrm{R}_{b} \mathrm{R}_{c} \mathrm{P}_{1} \mathrm{R}_{c}$ can be obtained from $\mathrm{R}_{a} \mathrm{R}_{b} \mathrm{R}_{c} \mathrm{P}_{1} \mathrm{P}_{2}$ by replacing $\mathrm{P}_{2}$ with $\mathrm{R}_{c}$. According to Eqs. (7) and (8), $\boldsymbol{S}_{f, \mathrm{~L}_{\mathrm{I}}}^{\prime}$ generated by $\mathrm{R}_{a} \mathrm{R}_{b} \mathrm{R}_{c} \mathrm{P}_{1} \mathrm{R}_{c}$ is

$$
\begin{aligned}
\boldsymbol{s}_{f, \mathrm{~L}_{\mathrm{I}}}^{\prime}= & 2 \tan \frac{\theta_{c}^{\prime}}{2}\left(\begin{array}{c}
\boldsymbol{s}_{c} \\
\boldsymbol{r}_{Q} \times \boldsymbol{s}_{c}
\end{array}\right) \Delta t_{1}\left(\begin{array}{c}
\mathbf{0} \\
\boldsymbol{s}_{1}
\end{array}\right) \Delta 2 \tan \frac{\theta_{c}}{2}\left(\begin{array}{c}
\boldsymbol{s}_{c} \\
\boldsymbol{r}_{O} \times \boldsymbol{s}_{c}
\end{array}\right) \\
& \Delta 2 \tan \frac{\theta_{b}}{2}\left(\begin{array}{c}
\boldsymbol{s}_{b} \\
\boldsymbol{r}_{O} \times \boldsymbol{s}_{b}
\end{array}\right) \Delta 2 \tan \frac{\theta_{a}}{2}\left(\begin{array}{c}
\boldsymbol{s}_{a} \\
\boldsymbol{r}_{O} \times \boldsymbol{s}_{a}
\end{array}\right) .
\end{aligned}
$$

Using the properties of screw triangle product, Eq. (19) can be rewritten as

$$
\begin{aligned}
\boldsymbol{S}_{f, \mathrm{~L}_{\mathrm{I}}}^{\prime} & =2 \tan \frac{\theta_{c}+\theta_{c}^{\prime}}{2}\left(\begin{array}{c}
\boldsymbol{s}_{c} \\
\boldsymbol{r}_{Q} \times \boldsymbol{s}_{c}
\end{array}\right) \\
& \Delta\left(\begin{array}{c}
\mathbf{0} \\
\left(\exp \left(\theta_{c} \tilde{s}_{c}\right)-\boldsymbol{E}_{3}\right)\left(\boldsymbol{r}_{Q}-\boldsymbol{r}_{O}\right)
\end{array}\right) \Delta t_{1}\left(\begin{array}{c}
\mathbf{0} \\
\exp \left(\theta_{c} \tilde{s}_{c}\right) \boldsymbol{s}_{1}
\end{array}\right) \\
& \Delta 2 \tan \frac{\theta_{b}}{2}\left(\begin{array}{c}
\boldsymbol{s}_{b} \\
\boldsymbol{r}_{O} \times \boldsymbol{s}_{b}
\end{array}\right) \Delta 2 \tan \frac{\theta_{a}}{2}\left(\begin{array}{c}
\boldsymbol{s}_{a} \\
\boldsymbol{r}_{O} \times \boldsymbol{s}_{a}
\end{array}\right),
\end{aligned}
$$

where $\tilde{\boldsymbol{s}}_{c}$ is the skew matrix of $\boldsymbol{s}_{c}, \boldsymbol{E}_{3}$ is a unit matrix of order three.

Because $\boldsymbol{s}_{c}^{\mathrm{T}} \boldsymbol{s}_{1}=0$, the second and third factors are two translations perpendicular to $s_{c}$. Using the similar derivations from Eq. (15) to Eq. (17), the following equivalent expression of Eq. (20) can be obtained

$$
\begin{aligned}
\boldsymbol{S}_{f, \mathrm{~L}_{\mathrm{I}}}^{\prime} & =2 \tan \frac{\theta_{c}+\theta_{c}^{\prime}}{2}\left(\begin{array}{c}
\boldsymbol{s}_{c} \\
\boldsymbol{r}^{\prime \prime}{ }_{c} \times \boldsymbol{s}_{c}
\end{array}\right) \Delta 2 \tan \frac{\theta_{b}}{2}\left(\begin{array}{c}
\boldsymbol{s}_{b} \\
\boldsymbol{r}_{O} \times \boldsymbol{s}_{b}
\end{array}\right) \\
& \triangle 2 \tan \frac{\theta_{a}}{2}\left(\begin{array}{c}
\boldsymbol{s}_{a} \\
\boldsymbol{r}_{O} \times \boldsymbol{s}_{a}
\end{array}\right),
\end{aligned}
$$

where

$$
\begin{array}{r}
\boldsymbol{r}^{\prime \prime}{ }_{c}=\boldsymbol{r}_{Q}-\frac{\left(t_{1} \exp \left(\theta_{c} \tilde{\boldsymbol{s}}_{c}\right) \boldsymbol{s}_{1}+\left(\exp \left(\theta_{c} \tilde{\boldsymbol{s}}_{c}\right)-\boldsymbol{E}_{3}\right)\left(\boldsymbol{r}_{Q}-\boldsymbol{r}_{O}\right)\right) \times \boldsymbol{s}_{c}}{2 \tan \frac{\theta_{c}+\theta_{c}^{\prime}}{2}}+ \\
\frac{t_{1} \exp \left(\theta_{c} \tilde{\boldsymbol{s}}_{c}\right) \boldsymbol{s}_{1}+\left(\exp \left(\theta_{c} \tilde{\boldsymbol{s}}_{c}\right)-\boldsymbol{E}_{3}\right)\left(\boldsymbol{r}_{Q}-\boldsymbol{r}_{O}\right)}{2} .
\end{array}
$$

For the same reason as discussed about $\boldsymbol{r}_{c}$ and $\boldsymbol{r}_{c}^{\prime}, \boldsymbol{r}^{\prime \prime}{ }_{c}$ denotes arbitrary vector which is perpendicular to $\boldsymbol{s}_{c}$, because $\left(t_{1} \exp \left(\theta_{c} \tilde{\boldsymbol{s}}_{c}\right) \boldsymbol{s}_{1}+\left(\exp \left(\theta_{c} \tilde{\boldsymbol{s}}_{c}\right)-\boldsymbol{E}_{3}\right)\left(\boldsymbol{r}_{Q}-\boldsymbol{r}_{O}\right)\right) \times \boldsymbol{s}_{c}$ and $t_{1} \exp \left(\theta_{c} \tilde{\boldsymbol{s}}_{c}\right) \boldsymbol{s}_{1}+\left(\exp \left(\theta_{c} \tilde{\boldsymbol{s}}_{c}\right)-\boldsymbol{E}_{3}\right)\left(\boldsymbol{r}_{Q}-\boldsymbol{r}_{O}\right)$ are two arbitrary orthogonal vectors that are perpendicular to $\boldsymbol{s}_{c}$. This means that Eq. (19) is equivalent to Eq. (13).

In this manner, $\mathrm{R}_{a} \mathrm{R}_{b} \mathrm{R}_{c} \mathrm{P}_{1} \mathrm{R}_{c}$ and $\mathrm{R}_{a} \mathrm{R}_{b} \mathrm{R}_{c} \mathrm{R}_{c} \mathrm{R}_{c}$ are synthesized as derivative limb structures.

Furthermore, it is easy to see that arbitrarily adjusting the sequence among two $\mathrm{R}_{c}$ and $\mathrm{P}_{1}$ will not change the generated finite screw based upon the derivations in the first situation. Hence, two additional derivative $\operatorname{limb}$ structures are obtained, i.e., $R_{a} R_{b} P_{1} R_{c} R_{c}$ and $\mathrm{R}_{a} \mathrm{R}_{b} \mathrm{R}_{c} \mathrm{R}_{c} \mathrm{P}_{1}$.

From the above analysis, totally seven 5 -DoF feasible limb structures for the 2T3R PKMs are synthesized. For simplicity, we rewrite the three adjacent $\mathrm{R}$ joints, $\mathrm{R}_{a} \mathrm{R}_{b} \mathrm{R}_{c}$, as $\mathrm{S}$, and the two adjacent $\mathrm{R}$ joints, $\mathrm{R}_{a} \mathrm{R}_{b}$, as $\mathrm{U}$ (universal joint). These seven limb structures are listed in Table 1. 
Having these limb structures at hand, we can obtain any 2T3R PKMs with several 5-DoF and 6-DoF limbs obeying some specific assembly conditions.

(1) According to Eq. (6), all the 5-DoF limbs in a 2T3R PKM should have the same translation plane and the same rotation center $O$. Thus, only one 5-DoF limb can be selected to compose a 2T3R PKM because $\mathrm{S}$ and/or $\mathrm{U}$ joints that belong to different limbs cannot be placed at a common point for the convenience of mechanical design.

(2) In order to design PKMs with suitable actuations, we select one 5-DoF limb and four 6-DoF limbs to compose a 2T3R PKM. In this way, each limb has one actuation. The four 6-DoF limbs are separated into two groups, which are placed symmetrically with respect to the translation plane of the 5-DoF limb. The five limbs are fixed at an icosahedron shape base in order to minimize risk of collisions and guarantee rigidity of the entire machine [37].

Using these assembly conditions, many innovative 2T3R PKMs can be synthesized. Here, we only list four topical PKMs due to space limitations, as shown in Figure 4. Some of these PKMs have been successfully applied in machining and manufacture or have great potential industrial applications because of their outstanding capabilities to realize large workspaces and flexible orientations, among which the SPR-4(SPRR) in Figure 4(a) is known as METROM PKM [37] and has been developed as commercial product by German company [37] (Additional files 2, 3, 4, 5).

\section{Kinematic Analysis Based upon Instantaneous Screws}

4.1 Parametric Model of a PKM

For a PKM composed of $l$ limbs as shown in Figure 3, its topological model has been formulated in Eqs. (6)-(8) based upon finite screws. As discussed in Section 2, the parametric model for kinematic analysis of the PKM can be directly formulated using the differential mapping between finite and instantaneous screws. According to Eq. (5), the instantaneous screws generated by the PKM, its limbs and joints can be obtained through differentiating the corresponding finite screws of them.

Firstly, the instantaneous screw generated by the $k$ th joint in the $i$ th limb at its initial pose can be obtained through differentiating Eq. (8) as

Table 1 5-DoF limb structures for 2T3R PKM

\begin{tabular}{ll}
\hline Standard limbs & Derivative limbs \\
\hline$S P_{1} P_{2}$ & $U P_{1} P_{2} R_{c^{\prime}} U P_{1} R_{c} P_{2}$ \\
& $S P_{1} R_{c^{\prime}} S R_{c} R_{c}$ \\
& $U P_{1} R_{c} R_{c^{\prime}} S R_{c} P_{1}$ \\
\hline
\end{tabular}

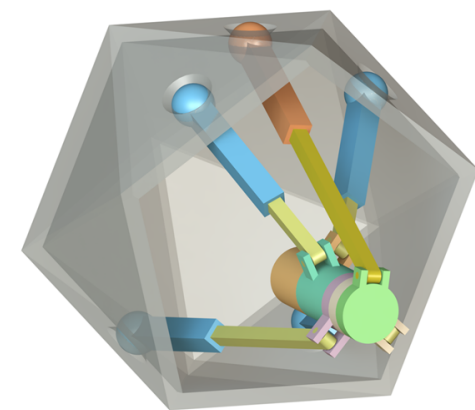

(a)

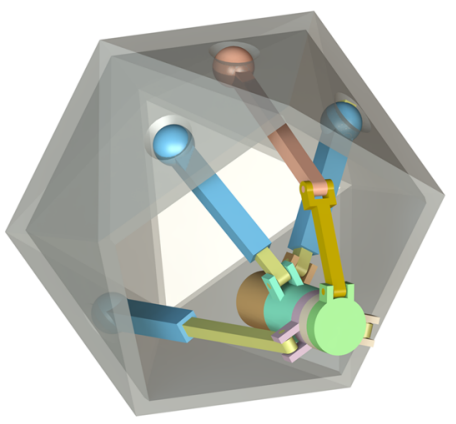

(b)

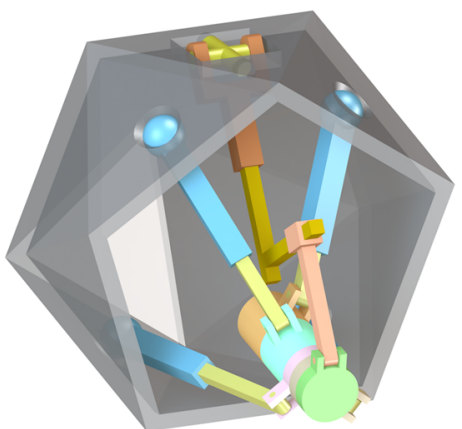

(c)

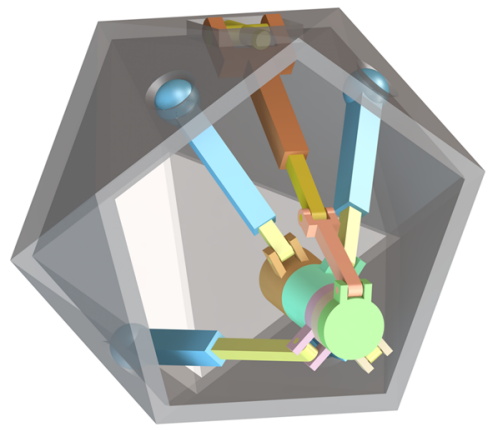

(d)

Figure 4 Typical 2T3R PKMs. a SPR-4(SPRR), b SRR-4(SPRR), c UPPR-

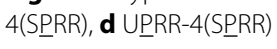




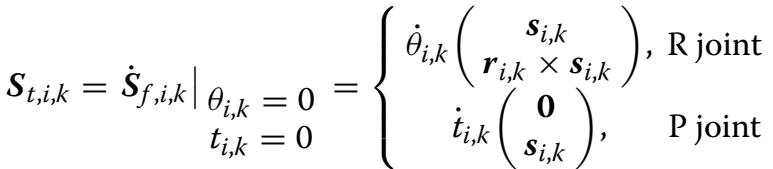

$$
\begin{aligned}
& =\left\{\begin{array}{cc}
\omega_{i, k}\left(\begin{array}{c}
\boldsymbol{s}_{i, k} \\
\boldsymbol{r}_{i, k} \times \boldsymbol{s}_{i, k}
\end{array}\right), & \mathrm{R} \text { joint } \\
v_{i, k}\left(\begin{array}{c}
\mathbf{0} \\
\boldsymbol{s}_{i, k}
\end{array}\right), & \text { P joint }
\end{array}\right.
\end{aligned}
$$

Equation (22) shows the velocity of each one-DoF joint in the PKM. It can serve as the parametric models of the joints by taking $\omega_{i, k}$ and $v_{i, k}$ as the parameters, because the unit and position vectors of the joints at their initial poses are determinate quantities.

In the similar manner, parametric model of the $i$ th limb can be obtained by differentiating Eq. (7) and obtaining the instantaneous screws of the limb at its initial pose

$$
\begin{aligned}
& \boldsymbol{S}_{t, i}=\left.\dot{\boldsymbol{S}}_{f, i}\right|_{\theta_{i, k}=0}{ }_{t_{i, k}=0}, k=1,2, \cdots, n_{i} \\
&=\left.\sum_{k=1}^{n_{i}} \dot{\boldsymbol{S}}_{f, i, k}\right|_{\theta_{i, k}=0}{ }_{t_{i, k}=0}, k=1,2, \cdots, n_{i} \\
&=\sum_{k=1}^{n_{i}} \boldsymbol{S}_{t, i, k} .
\end{aligned}
$$

Finally, the parametric model of the PKM can be formulated through differentiating Eq. (6) as

$$
\begin{aligned}
& \boldsymbol{S}_{t, \mathrm{PKM}}=\left.\dot{\boldsymbol{S}}_{f, \mathrm{PKM}}\right|_{\theta_{i, k}=0} \quad i=1,2, \cdots, l \\
& t_{i, k}=0^{\prime} k=1,2, \cdots, n_{i}
\end{aligned}
$$

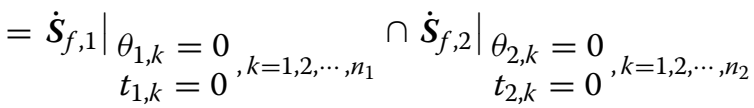

$$
\begin{aligned}
& \begin{aligned}
\left.\cap \cdots \cap \dot{\boldsymbol{S}}_{f, l}\right|_{\theta_{l, k}} & =0 \\
t_{l, k} & =0 \\
, k=1,2, \cdots, n_{l} &
\end{aligned} \\
& =\boldsymbol{S}_{t, 1} \cap \boldsymbol{S}_{t, 2} \cap \cdots \cap \boldsymbol{S}_{t, l} \text {. }
\end{aligned}
$$

From Eqs. (22)-(24), it can be clearly seen that the relationships between the parametric models of a PKM, its limbs and joints obtained in this paper are coincident with those given by other traditional approaches. However, unlike the traditional approaches, it is unnecessary to obtain the instantaneous screw system of a PKM through solving intersection of the instantaneous screw systems of its limbs in our approach. Based upon Eq. (24), the instantaneous screw system of the PKM can be directly formulated through differentiating the finite screw given in its topological model. In this way, the Jacobian matrix of the PKM for velocity, force, precision and stiffness modeling can be easily carried out using the obtained instantaneous screw system, which is ready to conduct kinematic analysis for parameter optimization and performance evaluation.

\subsection{Kinematic Analysis of METROM PKM}

Taking a typical structure of the synthesized innovative PKMs in Section 3, i.e., S $\underline{P} R-4(S \underline{P} R R)$ (METROM) PKM shown in Figure 4(a), for example, the detailed procedures of how to directly obtain its parametric model through differentiating the topological model will be shown. The instantaneous screws related Jacobian matrix will then be formulated for kinematic analysis.

According to the derivations in Section 3, the topological model of the METROM PKM can be formulated as

$$
\begin{aligned}
\boldsymbol{S}_{f, \mathrm{METROM}} & =t_{2}\left(\begin{array}{c}
\mathbf{0} \\
\boldsymbol{s}_{2}
\end{array}\right) \Delta t_{1}\left(\begin{array}{c}
\mathbf{0} \\
\boldsymbol{s}_{1}
\end{array}\right) \Delta 2 \tan \frac{\theta_{c}}{2}\left(\begin{array}{c}
\boldsymbol{s}_{c} \\
\boldsymbol{r}_{O} \times \boldsymbol{s}_{c}
\end{array}\right) \\
& \triangle 2 \tan \frac{\theta_{b}}{2}\left(\begin{array}{c}
\boldsymbol{s}_{b} \\
\boldsymbol{r}_{O} \times \boldsymbol{s}_{b}
\end{array}\right) \triangle 2 \tan \frac{\theta_{a}}{2}\left(\begin{array}{c}
\boldsymbol{s}_{a} \\
\boldsymbol{r}_{O} \times \boldsymbol{s}_{a}
\end{array}\right) .
\end{aligned}
$$

Using the derivative properties of screw triangle product [33], the parametric model of this PKM can be directly obtained by taking differential of Eq. (25)

$$
\begin{aligned}
\boldsymbol{S}_{t, \mathrm{METROM}} & =\left.\dot{\boldsymbol{S}}_{f, \mathrm{METROM}}\right|_{\theta_{m}=0} \\
t_{n}=0 & , m=a, b, c, n=1,2 \\
& =\dot{t}_{2}\left(\begin{array}{c}
\mathbf{0} \\
\boldsymbol{s}_{2}
\end{array}\right)+\dot{t}_{1}\left(\begin{array}{c}
\mathbf{0} \\
\boldsymbol{s}_{1}
\end{array}\right)+\dot{\theta}_{c}\left(\begin{array}{c}
\boldsymbol{s}_{c} \\
\boldsymbol{r}_{O} \times \boldsymbol{s}_{c}
\end{array}\right) \\
& +\dot{\theta}_{b}\left(\begin{array}{c}
\boldsymbol{s}_{b} \\
\boldsymbol{r}_{O} \times \boldsymbol{s}_{b}
\end{array}\right)+\dot{\theta}_{a}\left(\begin{array}{c}
\boldsymbol{s}_{a} \\
\boldsymbol{r}_{O} \times \boldsymbol{s}_{a}
\end{array}\right) \\
& =v_{2}\left(\begin{array}{c}
\mathbf{0} \\
\boldsymbol{s}_{2}
\end{array}\right)+v_{1}\left(\begin{array}{c}
\mathbf{0} \\
\boldsymbol{s}_{1}
\end{array}\right)+\omega_{c}\left(\begin{array}{c}
\boldsymbol{s}_{c} \\
\boldsymbol{r}_{O} \times \boldsymbol{s}_{c}
\end{array}\right) \\
& +\omega_{b}\left(\begin{array}{c}
\boldsymbol{s}_{b} \\
\boldsymbol{r}_{O} \times \boldsymbol{s}_{b}
\end{array}\right)+\omega_{a}\left(\begin{array}{c}
\boldsymbol{s}_{a} \\
\boldsymbol{r}_{O} \times \boldsymbol{s}_{a}
\end{array}\right) .
\end{aligned}
$$

It means that the instantaneous screw generated by the METROM PKM at its initial pose is the linear combination of five instantaneous screws. Thus, the Jacobian matrix of this PKM can be obtained through rewriting Eq. (26) into matrix form

$$
\boldsymbol{S}_{t, \mathrm{METROM}}=\left[\begin{array}{ccccc}
\boldsymbol{s}_{a} & \boldsymbol{s}_{b} & \boldsymbol{s}_{c} & \mathbf{0} & \mathbf{0} \\
\boldsymbol{r}_{O} \times \boldsymbol{s}_{a} & \boldsymbol{r}_{O} \times \boldsymbol{s}_{b} & \boldsymbol{r}_{O} \times \boldsymbol{s}_{c} & \boldsymbol{s}_{1} & \boldsymbol{s}_{2}
\end{array}\right]\left[\begin{array}{c}
\omega_{a} \\
\omega_{b} \\
\omega_{c} \\
v_{1} \\
v_{2}
\end{array}\right] .
$$

Based upon this Jacobian matrix, the constraint force exerted on the moving platform of the PKM can be found. It is a line vector whose axis passes through point $O$ with the direction $\boldsymbol{s}_{1} \times \boldsymbol{s}_{2}$, which restrains the one-DoF translation along its direction. It is expressed by a screw (wrench) as 
$\boldsymbol{S}_{w, \mathrm{METROM}}=f\left(\begin{array}{c}\boldsymbol{s}_{3} \\ \boldsymbol{r}_{O} \times \boldsymbol{s}_{3}\end{array}\right), \boldsymbol{s}_{3}=\frac{\boldsymbol{s}_{1} \times \boldsymbol{s}_{2}}{\left|\boldsymbol{s}_{1} \times \boldsymbol{s}_{2}\right|}$.

Having the Jacobian matrix and constraint force of the METROM PKM at hand, kinematic analysis of it can be carried out. In this way, parameter optimization and performance evaluation can be done, which are important parts in the parameter innovative design of METROM PKM. Because velocity and force [24-26], precision [27, 28] and stiffness [29-32] analysis of PKMs using Jacobian matrix and constraint force under instantaneous screws are widely researched, we do not give the detailed procedures here due to space limitation.

\section{Conclusions}

This paper presents a finite and instantaneous screw based approach for topology design and kinematic analysis. A kind of 2T3R 5-axis PKMs is taken as example to show the validity of the proposed approach. Following conclusions are drawn.

(1) The topological models of PKMs are formulated by describing finite motions of the PKMs, their limbs and joints by finite screws.

(2) Using the derivative mapping between finite and instantaneous screws, the parametric models of PKMs are proved to be directly obtained by differentiating the corresponding topological models.

(3) Using these models, type synthesis and kinematic analysis of PKMs can be carried out and strongly related. Type synthesis for topology design and kinematic analysis for parameter optimization and performance evaluation of a kind of 2T3R 5-axis PKMs are done to show the validity of the proposed approach.

\section{Additional files}

Additional file 1. Brief Introduction to this paper.

Additional File 2. Vedio of Tricept IV parallel kinematic machine.

Additional File 3. Prototype of Tricept IV parallel kinematic machine.

Additional file 4. Prototype of PaQuad parallel robot.

Additional file 5. Simulation of PaQuad parallel robot.

\section{Authors' Contributions}

TS was in charge of the whole trial; TS and S-FY wrote the manuscript. All authors read and approved the final manuscript.

\footnotetext{
Author details

${ }^{1}$ Key Laboratory of Mechanism Theory and Equipment Design of Ministry of Education, Tianjin University, Tianjin 300350, China. ${ }^{2}$ School of Engineering, The University of Warwick, Coventry CV4 7AL, UK. ${ }^{3}$ Centre for Robotics Research, School of Natural Sciences and Mathematics, King's College London, University of London, London WC2R 2LS, UK.
}

Authors' Information

Tao Sun, born in 1983, is currently a professor at Tianjin University, China. He received his PhD degree from Tianjin University, China, in 2012. His research interests include type synthesis, kinematic/static/dynamic modeling, analysis and optimal design of parallel mechanisms under framework of screw theory, orthopedics medical robot. Tel: +86-22-87402015; E-mail: stao@tju.edu.cn.

Shuo-Fei Yang, born in 1988, is appointed as a research associate at The Hong Kong Polytechnic University, Hong Kong, China. He received his PhD degree from Tianjin University, China, in 2017. His research interests include type synthesis and kinematic analysis of parallel mechanisms.E-mail: yangsf@ tju.edu.cn.

Tian Huang, born in 1953, is currently a professor at Tianjin University, China. He received his PhD degree from Tianjin University, China, in 1990. His research interests include kinematics and dynamics of machine tools and robotics. E-mail: tianhuang@tju.edu.cn.

Jian S. Dai, born in 1954, is currently a professor at King's College London, University of London, UK. He received his PhD degree from University of Salford, UK, in 1993. His research interests include theoretical and computational kinematics, reconfigurable mechanisms, dexterous mechanisms and manipulators, end-effectors and multifingered hands. E-mail: jian.dai@kcl.ac.uk.

\section{Competing Interests}

The authors declare that they have no competing interests.

Ethics Approval and Consent to Participate

Not applicable.

Funding

Supported by National Natural Science Foundation of China (Grant No. 51675366), and Tianjin Research Program of Application Foundation and Advanced Technology (Grant Nos. 16JCYBJC19300, 15JCZDJC38900).

\section{Publisher's Note}

Springer Nature remains neutral with regard to jurisdictional claims in published maps and institutional affiliations.

Received: 22 June 2017 Accepted: 16 April 2018

Published online: 21 May 2018

\section{References}

[1] "Made in China 2025" Plan, Ministry of Industry and Information Technology of the People's Republic of China. http://www.miit.gov.cn. Accessed 22 May 2017.

[2] "Made in China 2025" Plan, Ministry of Science and Technology of the People's Republic of China. http://www.most.gov.cn. Accessed 22 May 2017.

[3] J He, F Gao, X D Meng, et al. Type synthesis for 4-DOF parallel press mechanism using $G_{F}$ set theory. Chinese Journal of Mechanical Engineering, 2015, 28(4): 851-859.

[4] F G Xie, X J Liu, Z You, et al. Type synthesis of 2T1R-type parallel kinematic mechanisms and the application in manufacturing. Robotics and Computer-Integrated Manufacturing, 2014, 30(1): 1-10.

[5] F Gao, W M Li, X C Zhao, et al. New kinematic structures for 2-, 3-, 4-, and 5-DOF parallel manipulator designs. Mechanism and Machine Theory, 2002, 37(11): 256-269.

[6] J S Dai, T S Zhao. Stiffness characteristics and kinematics analysis of twolink elastic underactuated manipulators. Journal of Robotic Systems, 2002, 19(4): 169-176.

[7] KT Zhang, J S Dai, Y F Fang. Constraint analysis and bifurcated motion of the 3PUP parallel mechanism. Mechanism and Machine Theory, 2012, 49: 256-269.

[8] X Li, X L Ding, G S Chirikjian. Analysis of a mechanism with redundant drive for antenna pointing. Proceedings of the Institution of Mechanical Engineers, Part G: Journal of Aerospace Engineering, 2017, 231(2): 229-239.

[9] B Li, W Zhao, Z Q Deng. Modeling and analysis of a multi-dimensional vibration isolator based on the parallel mechanism. Journal of Manufacturing Systems, 2012, 31(1): 50-58. 
[10] F Jiang, H S Ding, T Fu, et al. The kinematic influence coefficient analysis of a parallel stabilized platform testing system. Advanced Materials Research, 2011, 308-310: 2120-2126.

[11] F Gao, W Z Guo, Q Y Song, et al. Current development of heavy-duty manufacturing equipments. Journal of Mechanical Engineering, 2010, 46(19): 92-107.

[12] F Gao. Reflection on the current status and development strategy of mechanism research. Journal of Mechanical Engineering, 2005, 41 (8): 3-17. (in Chinese).

[13] LW Tsai. Robot analysis: the mechanics of serial and parallel manipulators. New York: John Wiley \& Sons, Inc., 1999.

[14] F Gao, J Yang, Q J Ge. GF set theory for parallel mechanism type synthesis. Beijing: Science Press, 2011.

[15] Z Huang, Q C Li, H F Ding. Theory of parallel mechanisms. Netherlands: Springer, 2013.

[16] J M Hervé. Analyse structurelle des mécanismes par groupe des déplacements. Mechanism and Machine Theory, 1978, 13(4): 437-450.

[17] J M Hervé, F Sparacino. Structural synthesis of "parallel" robots generating spatial translation. Proceedings of the IEEE International Conference on Advanced Robotics, Pisa, Italy, 1991: 808-813.

[18] Q C Li, J M Hervé. Parallel mechanisms with bifurcation of schoenflies motion. IEEE Transactions on Robotics, 2009, 25(1): 158-164.

[19] Q C Li, J M Hervé. Type synthesis of 3-DOF RPR-equivalent parallel mechanisms. IEEE Transactions on Robotics, 2014, 30(6): 1333-1343.

[20] R W Brockett. Mathematical theory of networks and systems. Robotic manipulators and the product of exponential formula, New York: Springer, 1984: 120-129.

[21] A Perez, J M McCarthy. Dual quaternion synthesis of constrained robotic systems. ASME Journal of Mechanical Design, 2004, 126(3): 425-435.

[22] J M Selig. Exponential and cayley maps for dual quaternions. Advances in Applied Clifford Algebras, 2010, 20(3): 923-936.

[23] J S Dai. Euler-Rodrigues formula variations, quaternion conjugation and intrinsic connections. Mechanism and Machine Theory, 2015, 92: 144-152.

[24] H B Qu, Y F Fang, S Guo. Theory of degrees of freedom for parallel mechanisms with three spherical joints and its applications. Chinese Journal of Mechanical Engineering, 2015, 28(4): 737-746.

[25] T Sun, Y M Song, G Dong. Optimal design of a parallel mechanism with three rotational degrees of freedom. Robotics and Computer-Integrated Manufacturing, 2012, 28(4): 500-508.

[26] J S Wang, CWu, X J Liu. Performance evaluation of parallel manipulators: motion/force transmissibility and its index. Mechanism and Machine Theory, 2010, 45(10): 1462-1476.

[27] T Sun, Y P Zhai, Y M Song. Kinematic calibration of a 3-DoF rotational parallel manipulator using laser tracker. Robotics and Computer-Integrated Manufacturing, 2016, 41: 78-91.
[28] S H Hu, X L Liu, J C Sun. Error modeling and simulation of serial-parallel mechanism based on screw theory. Applied Mechanics and Materials, 2014, 527: 156-161.

[29] T Sun, B B Lian, Y M Song. Stiffness analysis of a 2-DoF over-constrained RPM with an articulated traveling platform. Mechanism and Machine Theory, 2016, 96(1): 165-178.

[30] Y Q Zhao, Y Jin, J Zhang. Kinetostatic modeling and analysis of an exechon parallel kinematic machine(PKM) module. Chinese Journal of Mechanical Engineering, 2016, 29(1): 33-44.

[31] B B Lian, T Sun, Y M Song. Stiffness analysis and experiment of a novel 5-DoF parallel kinematic machine considering gravitational effects. International Journal of Machine Tools and Manufacture, 2015, 95: 82-96.

[32] J J Yu, S S Bi, G H Zong. Analysis for the static stiffness of a 3-DOF parallel compliant micromanipulator. Journal of Mechanical Engineering, 2002, 38(4): 7-10. (in Chinese).

[33] T Sun, S F Yang, T Huang, et al. A way of relating instantaneous and finite screws based on the screw triangle product. Mechanism and Machine Theory, 2017, 108: 75-82.

[34] S F Yang, T Sun, T Huang, et al. A finite screw approach to type synthesis of three-DOF translational parallel mechanisms. Mechanism and Machine Theory, 2016, 104: 405-419.

[35] S F Yang, T Sun, T Huang. Type synthesis of parallel mechanisms having 3T1R motion with variable rotational axis. Mechanism and Machine Theory, 2017, 109: 220-230.

[36] T Sun, S F Yang, T Huang, et al. A generalized and analytical method to solve inverse kinematics of serial and parallel mechanisms using finite screw theory. Proceedings of 7th IFTOMM International Workshop on Computational Kinematics (IFTOMM CK 2017), Poitiers, France, May 22-24, 2017 : 602-608.

[37] Mobile 5-Achs-Fräsmaschine mit Rundtisch, METROM Mechatronische Maschinen $\mathrm{GmbH}$. http://www.metrom-mobil.com. Accessed 22 May 2017.

[38] F G Xie, X J Liu, X Luo. Mobility, singularity, and kinematics analyses of a novel spatial parallel mechanism. ASME Journal of Mechanisms and RobotiCs, 2016, 8(6): 061022-1-061022-10.

[39] X L Ding, J M Selig, J S Dai. Screw theory on the analysis of mechanical systems with spatial compliant. Journal of Mechanical Engineering, 2005, 41(8): 63-68. (in Chinese)

[40] Y Lu, B Hu. Analyzing kinematics and solving active/constrained forces of a 3SPU + UPR parallel manipulator. Mechanism and Machine Theory, 2007, 42(10): 1298-1313.

[41] C X Tian, Y F Fang, S Guo. Structural synthesis of a class of 2R2T hybrid mechanisms. Chinese Journal of Mechanical Engineering, 2016, 29(4): 703-709.

\section{Submit your manuscript to a SpringerOpen ${ }^{\circ}$ journal and benefit from:}

- Convenient online submission

- Rigorous peer review

- Open access: articles freely available online

- High visibility within the field

Retaining the copyright to your article

Submit your next manuscript at springeropen.com 